Revista de Comunicación y Salud, 2018, Vol. 8, no 1, pp. 27-43

Editado por Cátedra de Comunicación y Salud

ISSN: 2173-1675

\title{
CONCIENTIZACIÓN EN LA SOCIEDAD DE CUENCA ACERCA DE LA DIABETES
}

\author{
Awareness in the society of Cuenca about the diabetes
}

\author{
Melina Jara \\ Universidad del Azuay
}

\begin{abstract}
Resumen
La presente investigación trata sobre la importancia del conocimiento de la diabetes en la sociedad cuencana, enfermedad que en este siglo gran parte de la población llega a padecer, causada por la falta de producción de insulina en el páncreas. Para ello se realizó una encuesta con el fin de conocer realmente cual era el conocimiento de la diabetes en la sociedad, obteniendo resultados bastante desfavorables, puesto que, la mayoría de los encuestados contestaban que no tenían conocimiento y además pensaban que ellos están libres de contraer esta enfermedad. Nadie está libre de ella, y es debido a la falta de información y formación, que mucha gente llega a padecerla. Con los datos obtenidos, se hará una campaña de educación y guía para todas aquellas personas que desconozcan de este tema y para que esta enfermedad no trascienda de generación en generación.
\end{abstract}

Palabras clave: prevención, educación, salud, diabetes.

\section{Abstract}

The present research deals with the importance of the knowledge of diabetes in the Cuenca society, a disease that in this century a large part of the population comes to suffer, caused by the lack of insulin production in the pancreas. To do this, a survey was conducted in order to really know what was the knowledge of diabetes in society, obtaining quite unfavorable results, since, most respondents answered that they had no knowledge and also thought that they are free to contract this sickness. Nobody is free of it, and it is due to the lack of information and training, that many people get to suffer it. With the data obtained, there will be an education and guidance campaign for all those people who do not know about this issue and so that this disease does not transcend from generation to generation.

Keywords: prevention, education, health, diabetes.

\section{Cómo citar el artículo}

Jara, M. (2018). Concientización en la sociedad de Cuenca acerca de la diabetes. Revista de Comunicación y Salud, 8(1), pp. 27-43.

DOI: http://doi.org/10.35669/revistadecomunicacionysalud.2018.8(1).27-43 


\section{INTRODUCCIÓN}

La diabetes es una enfermedad en la cual los niveles de glucosa en la sangre están por encima de lo normal. La mayor parte de los alimentos que consumimos se transforman en glucosa, o azúcar, que es utilizada por el cuerpo para generar energía. El páncreas, un órgano que se encuentra cerca del estómago, produce una hormona llamada insulina para facilitar el transporte de la glucosa a las células del organismo; cuando se sufre de diabetes, el cuerpo no produce suficiente insulina o no puede utilizar su propia insulina adecuadamente. Esto causa que el azúcar se acumule en la sangre (CDC. Centros para el Control y la Prevención de Enfermedades, 2015).

Los tres principales tipos de diabetes son: diabetes tipo 1, el cual es el resultado de un proceso autoinmune con un inicio muy repentino, necesitan terapia de insulina para sobrevivir; la diabetes tipo 2, por su parte, puede pasar inadvertida y sin diagnosticar durante años. En tales casos, los afectados no son conscientes del daño a largo plazo, y la diabetes gestacional, aparece durante el embarazo, puede conducir a graves riesgos para la salud de la madre y su bebé y aumentar el riesgo de desarrollar diabetes tipo 2 más tarde en la vida (Asociación Diabetes Madrid, 2015).

Esta dolencia puede producir complicaciones de salud graves tales como enfermedades cardiacas, ceguera, insuficiencia renal y amputaciones de las extremidades inferiores; la diabetes es la séptima causa principal de muerte en el mundo. Pero no solamente hay que encontrarle el punto malo a esta enfermedad puesto que es posible llevar un estilo de vida normal y saludable en el sentido de que se pueden probar nuevos alimentos, siendo esto es lo más probable, ya que cuando uno debuta en diabetes, los médicos recomiendan hacer algunos cambios significativos en la dieta.

En lugar de considerar el diagnóstico como el momento de eliminar de su menú aquellos alimentos que son favoritos, piense en la oportunidad de probar alimentos nuevos. No se trata de no comer pasteles, sino de probar pasteles para diabéticos, que los hay, o probar nuevos postres. Recomendamos hacer una lista con los nuevos alimentos a probar y que son buenos para la esta afección, degustando uno nuevo cada semana y no olvidar también que es la oportunidad de encontrar nuevos amigos. En esta sociedad cada vez mejor comunicada quizás la diabetes sea la oportunidad de contactar con alguno de los millones de personas que la padecen en el mundo y compartir las experiencias personales relacionadas. Se pueden encontrar grupos de apoyo, tanto reales como virtuales, donde compartir temores, preocupaciones, ideas, recetas y otros temas sobre la enfermedad; esto siempre enriquece. (Canala Diabetes, 2016).

Aprender a cocinar, aprender de qué manera esos alimentos y sus recetas afectan a nuestro metabolismo, puede servir para darle la vuelta a la sartén y experimentar en el maravilloso arte culinario, experimentar y probar es una manera de desarrollarse, ser disciplinado en estos hábitos llevan a otros y son también una motivación para hacer deporte o ejercicio. Necesitamos en muchas ocasiones un fin o un aliciente para movernos y la diabetes puede ser ese aspecto motivacional para ello. Somos conscientes que la realización de ejercicio beneficiará nuestro control de

Revista de Comunicación y Salud, 2018, Vol. 8, n 1, pp. 27-43 
la enfermedad, y por eso en la mayoría de las ocasiones dejaremos nuestro cómodo sofá por el paseo matinal en el parque.

Tener una vida más sana es lo que significa tomar conciencia de esta dolencia puesto que, si se siguen los consejos médicos, la dieta de pérdida de peso, el ejercicio, el manejo del estrés y se mantiene contacto con otros pacientes con diabetes, mejorará no sólo su control de la enfermedad, sino también su salud en general. Adoptará hábitos saludables que conseguirán que usted esté más sano, pero no sólo usted, su familia se aprovechará de sus nuevos hábitos y conseguirá un entorno a su alrededor más saludable, donde los modelos sociales que imperen sean los de una correcta alimentación y práctica de ejercicio. (Canala Diabetes, 2016).

La mayoría de médicos recomiendan todo lo dicho anteriormente, pues hay que cuidarse, especialmente en la comida, para prevenir cualquier síntoma de la diabetes, más aún si tenemos a nuestro alrededor familiares que lo posean, pues se dice que de cada familiar que tenga diabetes cuatro personas son propensas a heredarlo.

Mariano Salinas, experto en casos de diabetes, comenta que hay formas de ayudar a las personas que la sufren, ya que a veces nuestra buena intención puede llegar a ser un fastidio, cuando un ser querido es diagnosticado, es normal que surja el deseo de hacer todo lo posible por auxiliarlo en dicha transición, para ello, siempre debemos tomar en cuenta que uno puede ayudar, siempre y cuando tenga permiso para hacerlo.

La Dra. Sonia Gaztambide, presidenta de la Sociedad Española de Diabetes (SED) señala: "caminar regularmente durante al menos 30 minutos al día, por ejemplo, ha demostrado reducir el riesgo de diabetes tipo 2 en un 35-40\%. Esta prevención queda, en ocasiones, fuera del ámbito estrictamente sanitario: los medios de comunicación, las empresas de alimentación, los colegios, etc. pueden contribuir a dar este mensaje.

El Dr. Javier Salvador, director del Departamento de Endocrinología y Nutrición de la Clínica Universidad de Navarra y presidente de la Sociedad Española de Endocrinología y Nutrición (SEEN), aclara los principales retos en el cuidado del paciente con diabetes que, a su parecer se clasifican en tres aspectos: "prevención, pues es imprescindible actuar sobre los factores causales relacionados entre los que destaca la obesidad y el sedentarismo; diagnóstico precoz, lo que permite proceder cuanto antes para evitar el deterioro de la célula beta, las complicaciones y optimización del tratamiento, donde la individualización y el abordaje global adquieren una relevancia clave". (La Vanguardia, 2012).

De esta manera, se puede concluir que la diabetes es una enfermedad crónica que impide a la glucosa llegar a las diferentes células haciendo que se quede en la sangre; lo cual, da lugar a niveles anormalmente altos de azúcar, lo que a la larga provoca una serie de trastornos en diferentes órganos, si no se controla la enfermedad. Es cierto que no tiene cura, pero sí se controla; para ello, lo que se come y el ejercicio que se realice será muy importante. (Diabetes, Bienestar \& Salud, 2014). 


\section{OBJETIVO GENERAL}

- Concientizar a la sociedad sobre la diabetes.

\section{OBJETIVOS ESPECÍFICOS}

- Proteger la salud.

- Prevenir o retardar la aparición de la diabetes y las complicaciones de mayor prevalencia entre las poblaciones adulta y adulta mayor.

- Educar a la sociedad acerca de esta enfermedad y consecuencias.

\section{MATERIALES Y MÉTODOS}

Para la presente investigación se pretende usar una estrategia educativa y es la de transformar o crear nuevas conductas en los diferentes grupos sociales usando como herramienta, entrevistas a profesionales para poder obtener un eslogan 0 mensaje con el que la gente se sienta identificada, además de realizar encuestas para obtener información de cuan educada esta la gente acerca de este tema y la campaña para todos los grupos sociales que conforman la ciudad de Cuenca.

La campaña será difundida en medios masivos de comunicación además que se pretende hacer una campaña de publicidad en vallas, usando un marketing de guerrilla, realizando charlas en centros educativos, programas de radio para culminar en una feria donde la gente podrá ser invitada a conocer sobre el tema y donde estén profesionales del ámbito que podrán educar con una correcta información a la sociedad actual mencionando no solo las consecuencias sino también sus beneficios, realizando alianzas con diferentes instituciones dedicadas a este ámbito como "La Casa de la Diabetes" institución que proporcionara el material, profesionales la correcta información acerca de la misma.

De igual manera, se realizará la difusión de la normatividad vigente entre la comunidad médica y la población general desarrollando campañas de comunicación educativa dirigidas a la prevención de los factores de riesgo y control de la diabetes generando metodologías didácticas para garantizar aprendizajes significativos en las intervenciones educativas que realicen los Centros de Capacitación y de esta manera facilitar el acceso a la información y actualización en temas de interés al personal de salud involucrado y población en general.

También se planea promover la integración y participación del "educador en diabetes", dentro del sector Salud que contribuya a la atención integral de este padecimiento fomentando estilos de vida saludable que contemplan una alimentación adecuada, control de peso, actividad física y promoción de la reducción del consumo de alcohol y tabaco.

\subsection{Metodología}

Realizar una encuesta para saber qué conocimientos tienen aquellas personas acerca de la diabetes. Serían unas 40 encuestas en total, tomando en cuenta la muestra y segmentando por edad, nivel socioeconómico, nivel de estudio. Es 
importante conocer el concepto que tienen las personas acerca de la diabetes y saber qué tanto saben, para diseñar y saber comunicar el tipo de campaña que queremos abordar. Para tabular estas encuestas y los resultados, se utilizará el sistema Spss.

\section{Modelo de encuesta}

\section{Edad:}

Nivel socioeconómico:

$1000-2000 \quad 2000-3000 \quad 3000-4000$

Nivel de estudios:

Primaria Secundario Tercer Nivel Cuarto Nivel

1. Realiza ejercicio:

- Sí

- No

2. ¿Por qué?

- Verse bien

- Salud

3. De acuerdo a la anterior pregunta, si respondió sí, ¿Cuántas veces realiza ejercicio?

- Diario

- Dos veces por semana

- Tres veces por semana

4. ¿Cuántas horas al día hace ejercicio, si lo hace?

- 30 min. a 1 hora

- 1 a 2 horas

- 2 o más

5. En cuanto a alimentación ¿Cuántas veces come frutas y verduras?

- Diario

- Dos veces por semana

- Tres veces por semana

6. ¿Cuál de estas bebidas consume más?

- Agua

- Cola

- Jugos

- Bebidas con moderación (cerveza)

7. ¿Cuántas veces al año realiza pruebas de sangre y por qué?

- 1 al año

- 2 al año

- 3 al año 
- Nunca

8. Aproximadamente, ¿Cuál es su peso y estatura?

- Peso $\mathrm{kg}$

- Estatura

- No sé

9. ¿Conoce qué es la diabetes?

- ¿Sí_qué es?

- No

10. ¿Conoce a alguien cercano o familiar que la padece?

- Sí

- No

11. ¿Sabe si se puede prevenir la diabetes?

- Sí

- No

12. ¿Cree que en algún momento de su vida puede llegar a tener diabetes?

- Sí

- No

- Por qué

13. ¿Cree que sería factible realizar campaña de prevención sobre la diabetes?

- Sí

- No

- Por qué

14. ¿A qué tipo de gente cree que les beneficiaría más una campaña sobre diabetes?

- Adultos

- Madres de familia

- Universitarios

- Niños

- Todos 


\section{RESULTADOS}

Tabla cruzada Realiza ejercicio*¿Por qué?

Recuento

\begin{tabular}{lrrr|r} 
& \multicolumn{2}{c|}{ ¿Por qué? } & \multicolumn{1}{c}{} \\
& & Verse bien & \multicolumn{1}{c}{ Salud } & \multicolumn{2}{c}{ Total } \\
\hline $\begin{array}{l}\text { Realiza } \\
\text { ejercicio }\end{array}$ & Sí & 7 & 12 & 19 \\
\hline Total & & & 12 & 19 \\
\hline & & 7 & & \\
\hline
\end{tabular}

Resumen de procesamiento de casos

\begin{tabular}{l|l|l|l|l|r|r} 
& \multicolumn{2}{c}{ Válido } & \multicolumn{2}{c}{ Casos } & \multicolumn{2}{c}{ Total } \\
& N & Porcentaje & N & Porcentaje & N & Porcentaje \\
\hline Realiza ejercicio* & 19 & $47,5 \%$ & 21 & $52,5 \%$ & 40 & $100,0 \%$ \\
¿Por qué? & & & & & & \\
\hline
\end{tabular}

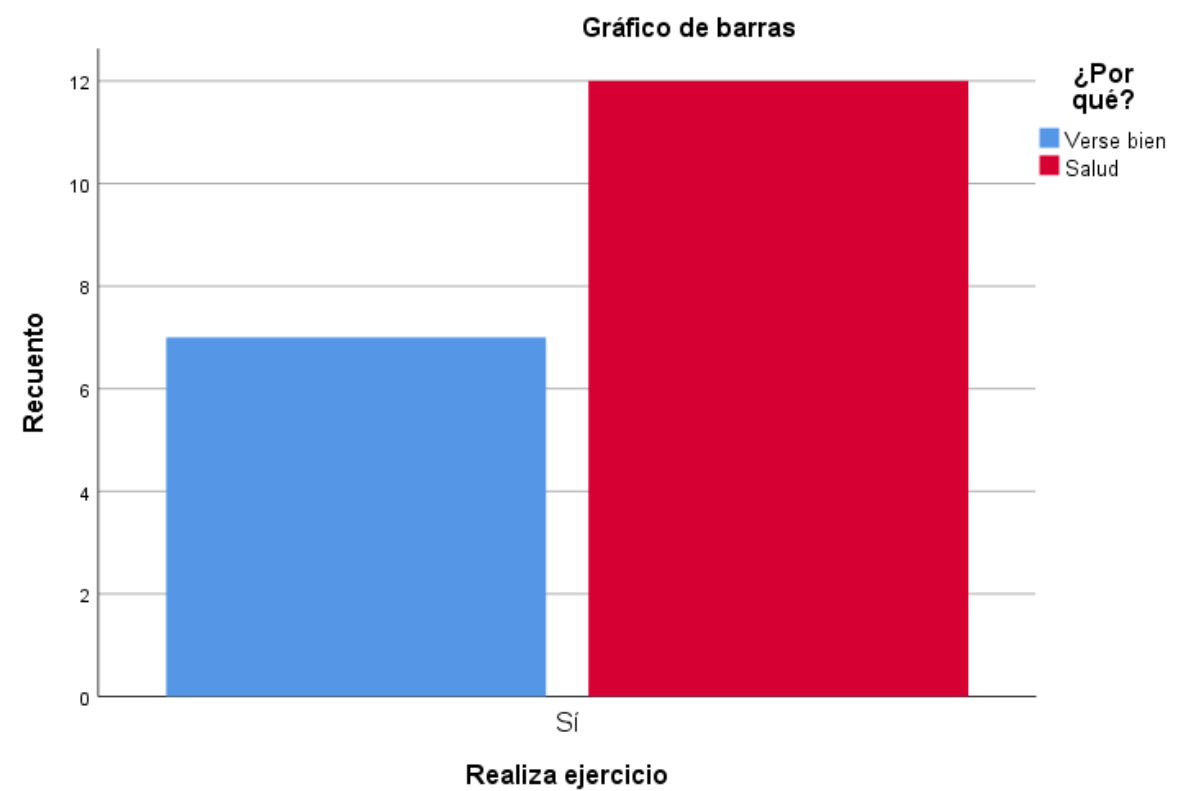

De las 40 personas encuestadas 19 personas realizan ejercicio de las cuales 7 lo hacen por verse bien y las 12 lo hacen por salud teniendo en cuenta que es un gran porcentaje quienes se preocupan más por su físico que por su salud. 


\begin{tabular}{|c|c|c|c|c|c|c|}
\hline \multicolumn{7}{|c|}{ Resumen de procesamiento de casos } \\
\hline & \multicolumn{6}{|c|}{ Casos } \\
\hline & \multicolumn{2}{|c|}{ Válido } & \multicolumn{2}{|c|}{ Perdido } & \multicolumn{2}{|c|}{ Total } \\
\hline & $\mathrm{N}$ & Porcentaje & $\mathrm{N}$ & Porcentaje & $\mathrm{N}$ & Porcentaje \\
\hline $\begin{array}{l}\text { Edad* ¿Cuántas } \\
\text { veces realiza } \\
\text { ejercicio? }\end{array}$ & 19 & $47,5 \%$ & 21 & $52,5 \%$ & 40 & $100,0 \%$ \\
\hline
\end{tabular}

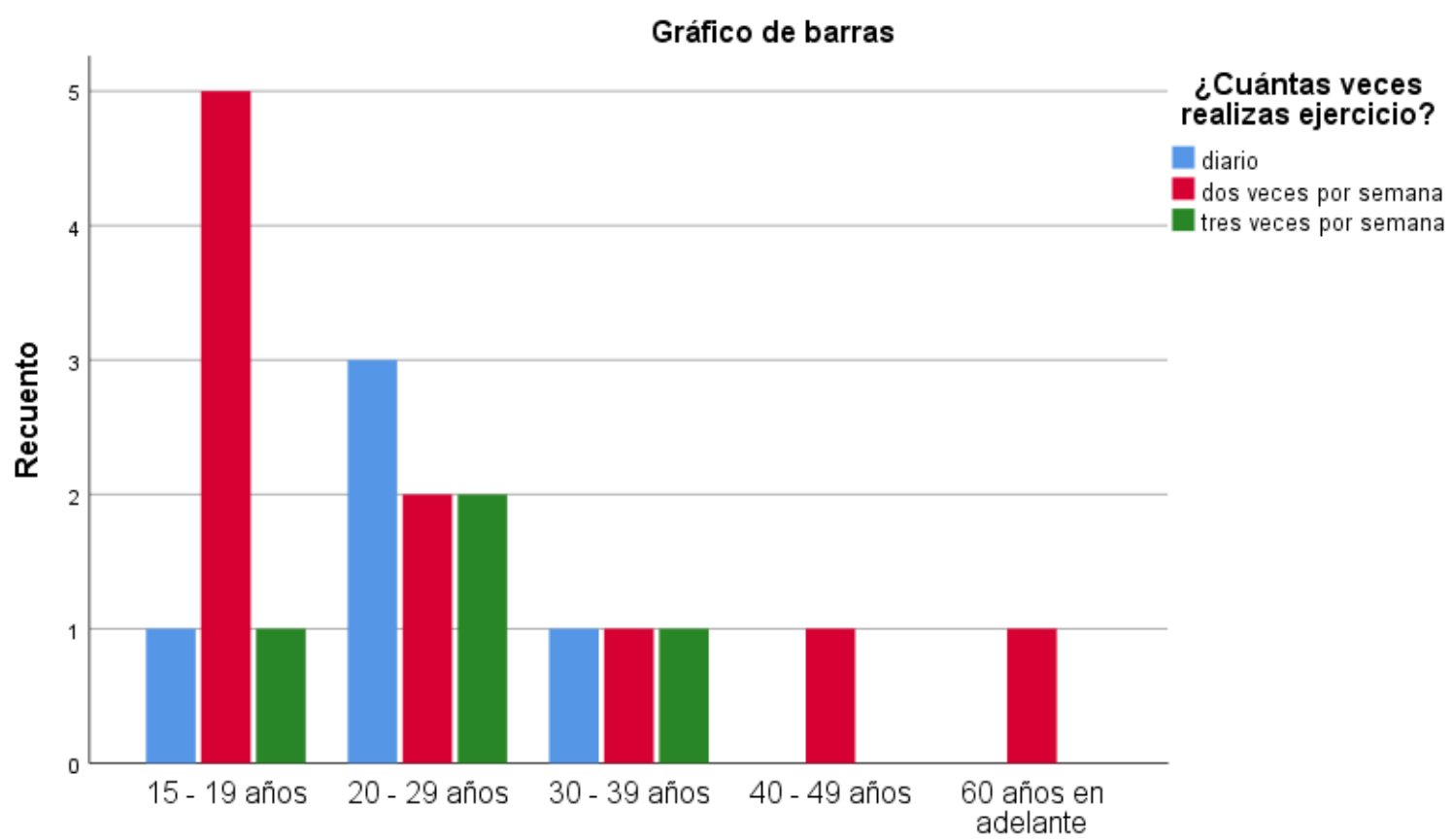

Edad

En la relación de las 19 personas que hacen ejercicio y sobre cuantas veces, se puede concluir según el gráfico, que las personas de 15 a 19 años realizan ejercicio dos veces por semana, mientras que las personas desde los 20 a 29 años lo hacen a diario, las personas de 30 a 39 años tienen un equilibro de ejercicio. Pese a estos resultados, nos damos cuenta de que no se ha perdido la costumbre de hacer ejercicio, aunque las personas de 20 a 29 años tienen más conciencia de eso.

\section{Resumen de procesamiento de casos}

Casos

\begin{tabular}{lc|c|c|c|c|c} 
& \multicolumn{2}{c|}{ Válido } & \multicolumn{2}{c|}{ Perdido } & \multicolumn{2}{c}{ Total } \\
& $\mathrm{N}$ & Porcentaje & $\mathrm{N}$ & Porcentaje & $\mathrm{N}$ & Porcentaje \\
\hline $\begin{array}{l}\text { Realiza ejercicio* } \\
\text { Horas de ejercicio }\end{array}$ & 19 & $47,5 \%$ & 21 & $52,5 \%$ & 40 & $100,0 \%$ \\
\hline
\end{tabular}


Tabla cruzada Realiza ejercicio *Horas de ejercicio Recuento

\begin{tabular}{lrr|r|r} 
& & \multicolumn{2}{c}{ Horas de ejercicio } & \\
& 1h-2h & 2h-más & \multicolumn{1}{c}{ Total } \\
\hline $\begin{array}{l}\text { Realiza } \\
\text { ejercicio }\end{array}$ & Sí & 10 & 9 & 19 \\
\hline Total & & & \\
\hline
\end{tabular}

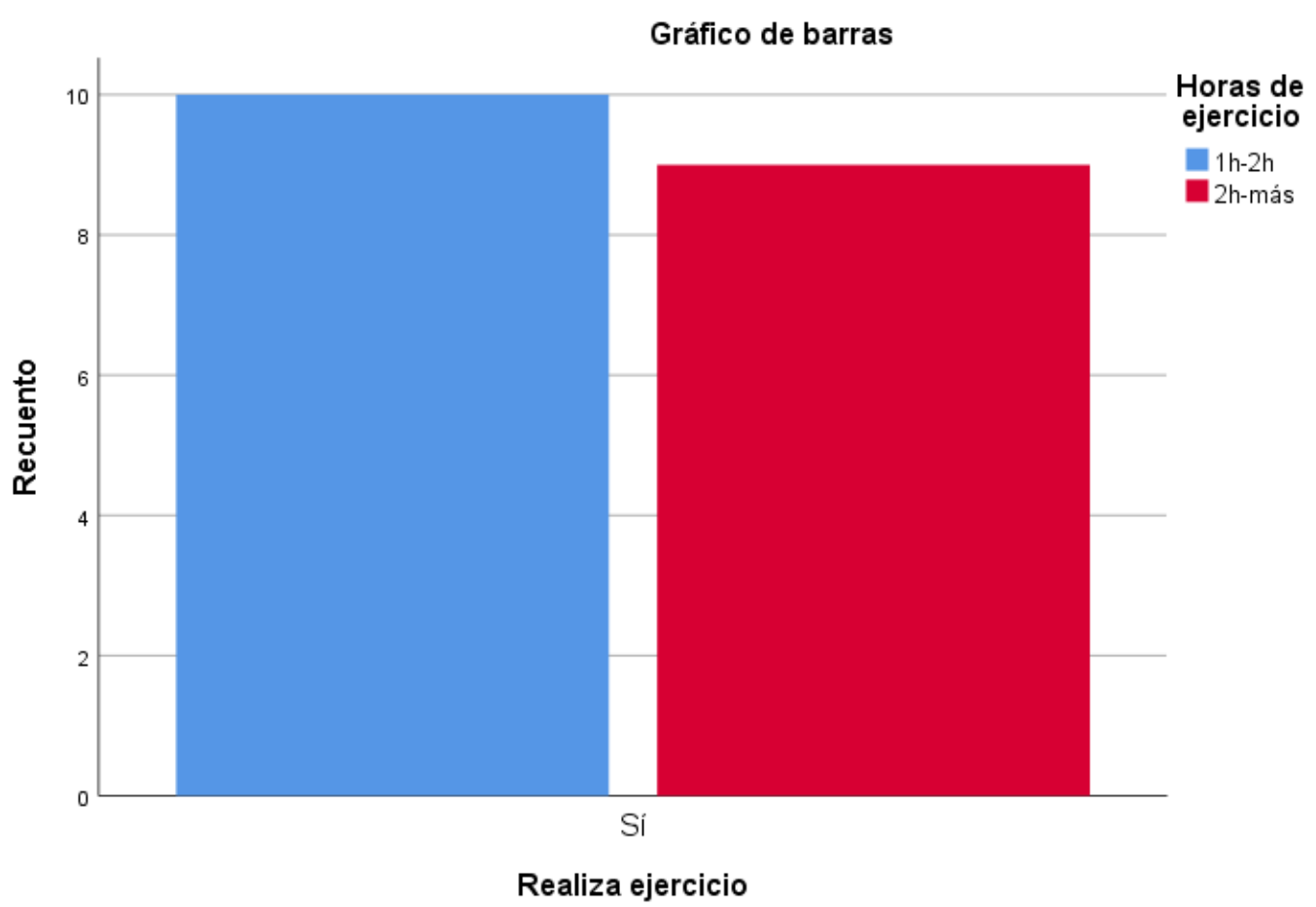

El $47.5 \%$ de los encuestados que realizan ejercicio están bajo el rango de ejercicio moderado de 1 hora a 2 horas.

Resumen de procesamiento de casos

\begin{tabular}{lc|c|c|c|c|c} 
& \multicolumn{2}{c}{ Válido } & \multicolumn{2}{c}{$\begin{array}{c}\text { Casos } \\
\end{array}$} & Perdido & \multicolumn{2}{c}{ Total } \\
& $\mathrm{N}$ & Porcentaje & $\mathrm{N}$ & Porcentaje & $\mathrm{N}$ & Porcentaje \\
\hline Edad $^{*}$ ¿Cuántas veces & 40 & $100,0 \%$ & 0 & $0,0 \%$ & 40 & $100,0 \%$ \\
come frutas y verduras? & & & & & & \\
\hline
\end{tabular}

Tabla cruzada Edad ¿¿Cuántas veces come frutas y verduras?

Recuento

\begin{tabular}{lrr|r|r|r|} 
& \multicolumn{6}{c}{ ¿Cuántas veces come frutas y verduras? } & \\
& diario & $\begin{array}{c}\text { 2 veces por } \\
\text { semana }\end{array}$ & $\begin{array}{c}\text { tres veces por } \\
\text { semana }\end{array}$ & Total \\
\hline Edad $\quad 15-19$ años & 8 & & 1 & & 1 \\
\hline
\end{tabular}

Revista de Comunicación y Salud, 2018, Vol. 8, no1, pp. 27-43 


\begin{tabular}{lr|r|r|r|r}
\hline $20-29$ años & 8 & 4 & 3 & 15 \\
\hline $30-39$ años & 5 & 4 & 1 & 10 \\
\hline $40-49$ años & 1 & 1 & 1 & 3 \\
\hline $\begin{array}{l}60 \text { años en } \\
\text { adelante }\end{array}$ & 2 & 0 & 0 & 2 \\
\hline Total & 24 & 10 & 6 & 40 \\
\hline
\end{tabular}

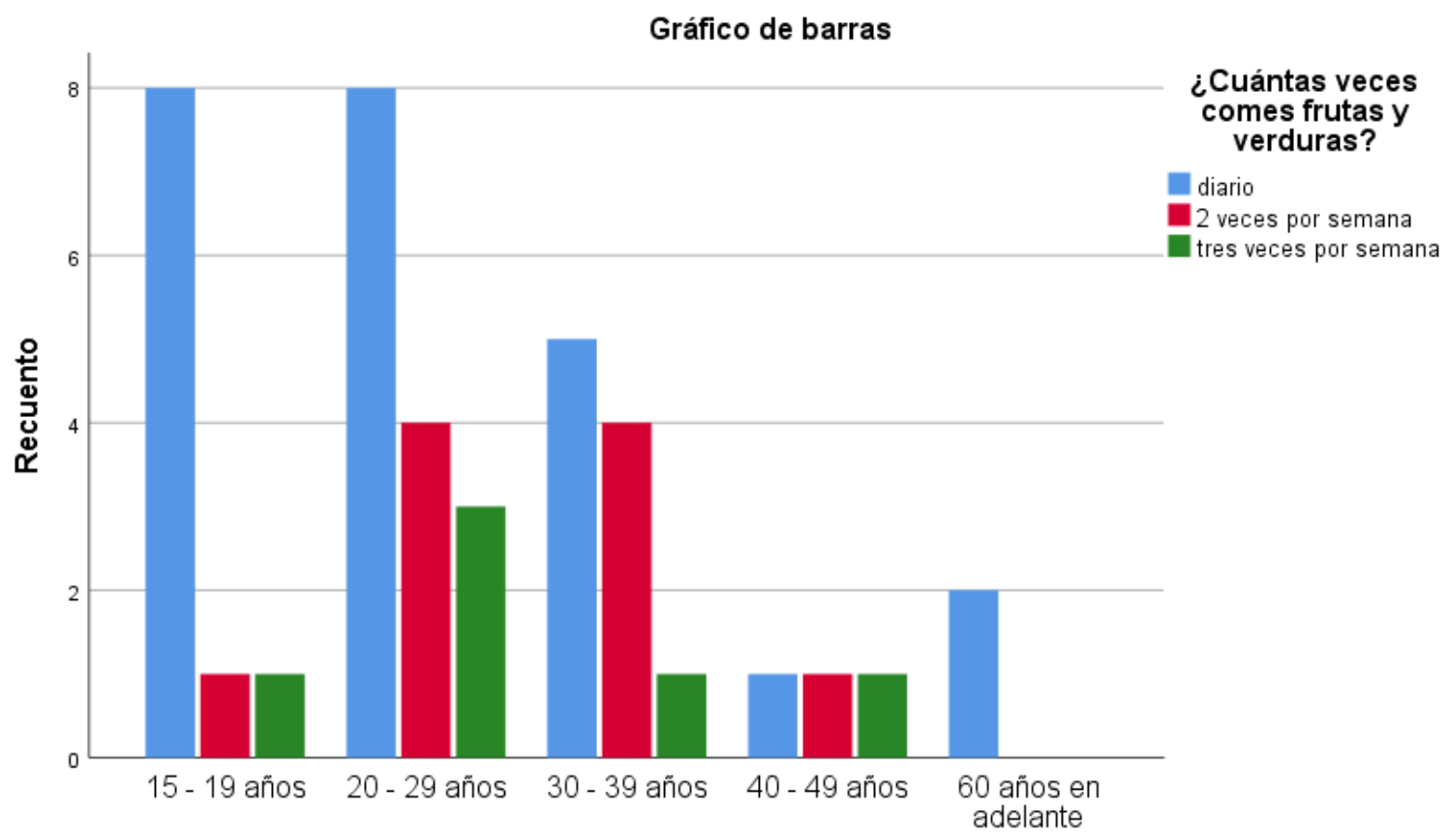

\section{Edad}

Pasando a los resultados de las variantes de alimentación el 100\% de los encuestados consumen frutas y verduras diariamente es decir no se ha perdido la buena costumbre de comer sano.

\section{Tabla cruzada Edad ¿QQué bebida consume más?}

Recuento

\begin{tabular}{|c|c|c|c|c|c|c|}
\hline & & & Ié bebid & consume & ás? & \\
\hline & & Agua & Cola & Jugos & $\begin{array}{c}\text { Con } \\
\text { moderación } \\
\text { (cerveza) }\end{array}$ & Total \\
\hline Edad & $15-19$ años & 6 & 0 & 4 & 0 & 10 \\
\hline & $20-29$ años & 6 & 0 & 7 & 2 & 15 \\
\hline & 30 - 39 años & 5 & 1 & 4 & 0 & 10 \\
\hline & 40 - 49 años & 1 & 0 & 2 & 0 & 3 \\
\hline & $\begin{array}{l}60 \text { años en } \\
\text { adelante }\end{array}$ & 2 & 0 & 0 & 0 & 2 \\
\hline Total & & 20 & 1 & 17 & 2 & 40 \\
\hline
\end{tabular}




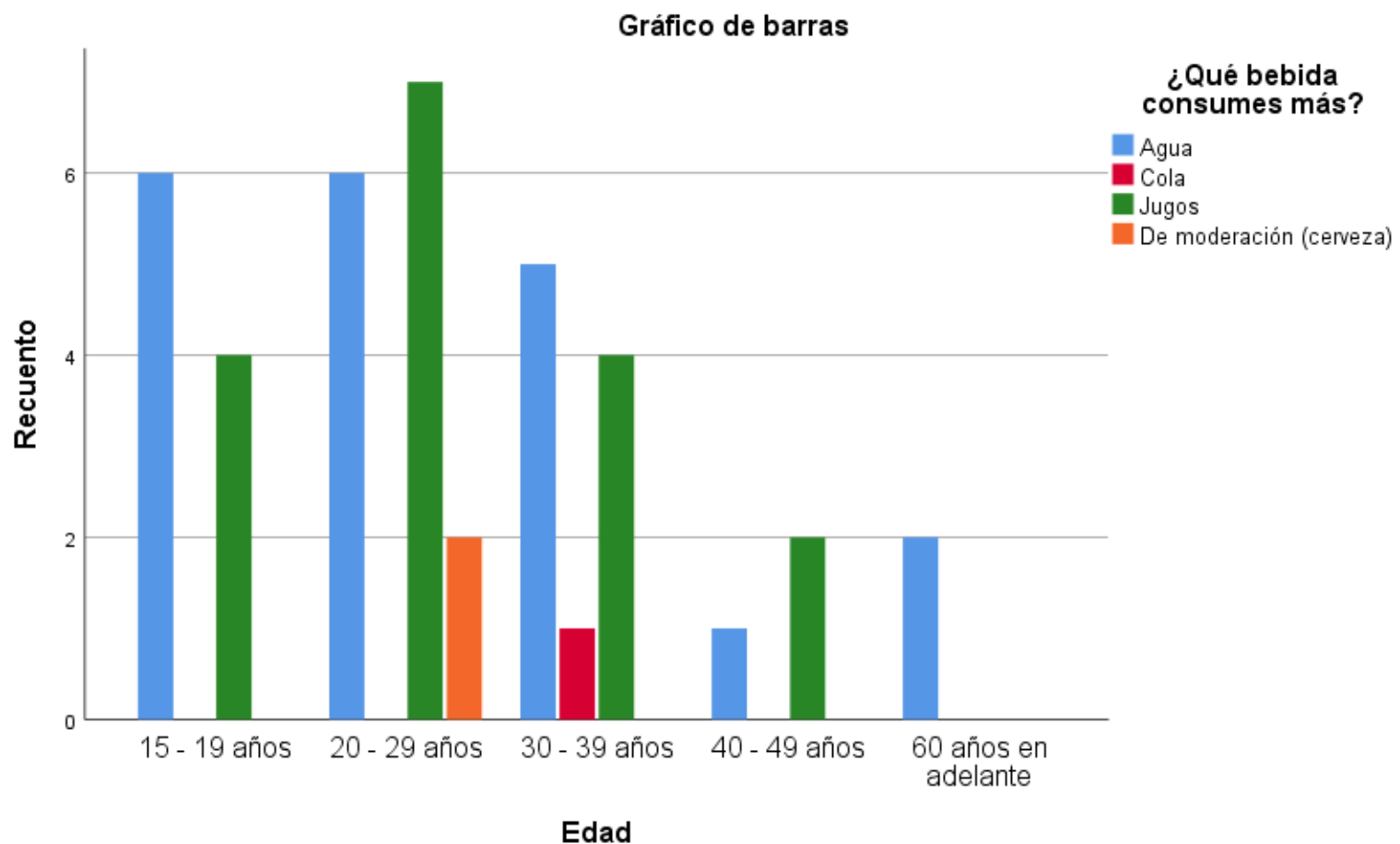

Resolviendo la pregunta sobre que bebida es la más consumida por los encuestados se llega a la conclusión de que los jóvenes de 15 a 19 años tiene un buen consumo de agua sin dejar muy atrás a los jugos, mientras que los encuestados de 20 a 29 años prefieren los jugos más que el agua; además de las bebidas de moderación en un porcentaje del $20 \%$. Las personas de 30 a 39 años prefieren el agua, pero al casi al mismo nivel que los jugos, además tienen un mal hábito de casi el 15\% de consumo de bebidas gaseosas. Desde los 40 a 49 años las personas pierden la costumbre de consumo de agua, siendo los jugos la bebida más consumida.

Tabla cruzada Edad¿Cuántas veces al año realiza pruebas de sangre? Recuento

¿Cuántas veces al año realiza pruebas de sangre?

\begin{tabular}{|c|c|c|c|c|c|c|}
\hline & & 1 al año & $2 \mathrm{al}$ año & 3 al año & Nunca & Total \\
\hline \multirow[t]{5}{*}{ Edad } & 15 - 19 años & 4 & 0 & 1 & 5 & 10 \\
\hline & 20 - 29 años & 7 & 2 & 1 & 5 & 15 \\
\hline & 30 - 39 años & 9 & 0 & 0 & 1 & 10 \\
\hline & 40 - 49 años & 1 & 1 & 0 & 1 & 3 \\
\hline & $\begin{array}{l}60 \text { años en } \\
\text { adelante }\end{array}$ & 1 & 0 & 1 & 0 & 2 \\
\hline Total & & 22 & 3 & 3 & 12 & 40 \\
\hline
\end{tabular}




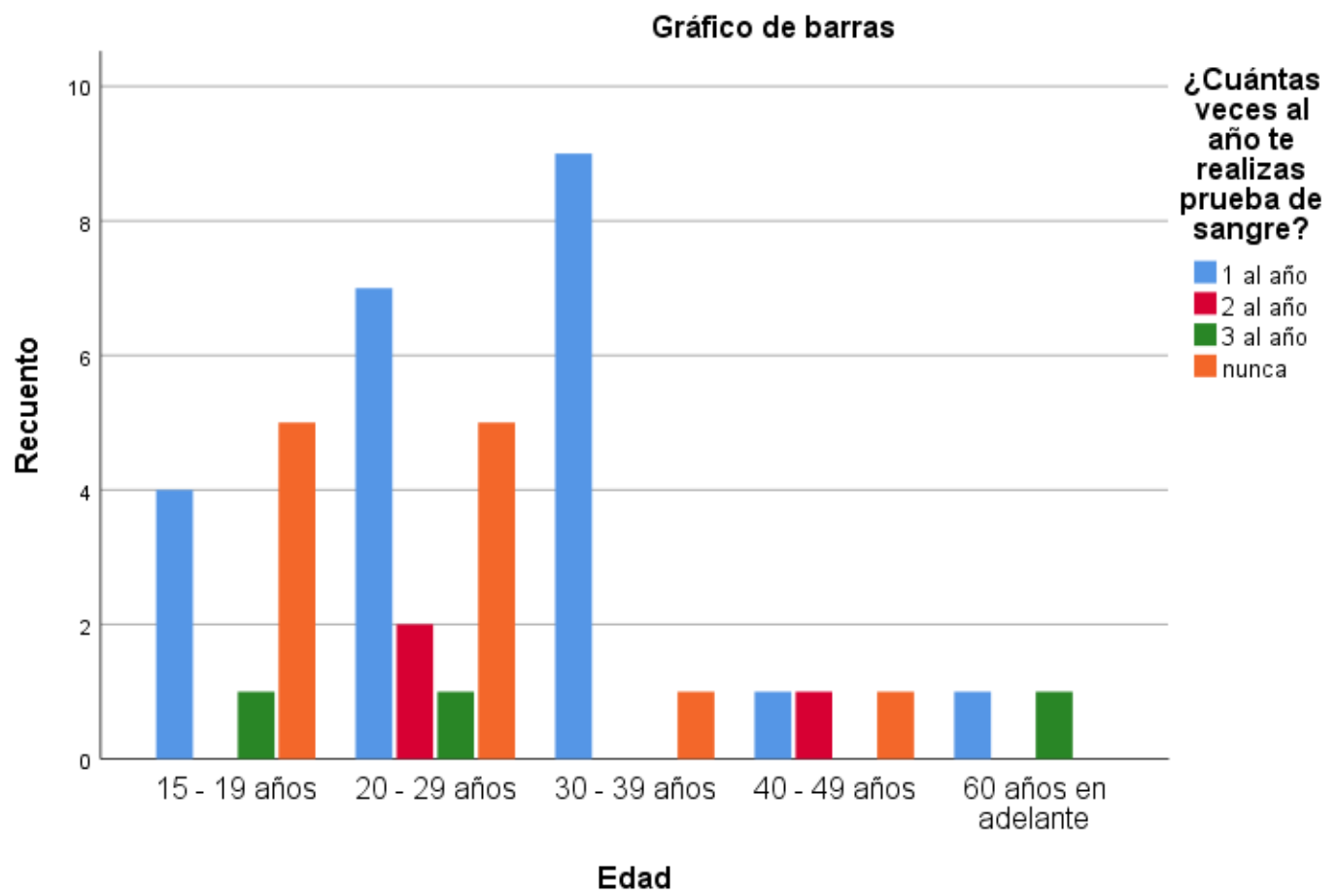

En cuanto a la preocupación por la salud y la realización de pruebas de sangre, las personas de 15 a 19 años no tienen preocupación por ello, siendo "nunca" la respuesta con un $40 \%$ por ciento de respuesta y una vez al año un $60 \%$. Esto no cambia en las personas de 20 a 29 años. Más delante, de 30 a 39 años ya empiezan a dar más importancia y el $80 \%$ se realiza exámenes una vez al año. De 40 años en adelante, existe ya una preocupación por la salud, teniendo en cuenta que ya se realizan exámenes de sangre por lo menos dos veces al año.

\section{Tabla cruzada Edad ${ }^{\star} ¿$ Conoce la diabetes?}

\begin{tabular}{|c|c|c|c|c|}
\hline \multicolumn{5}{|c|}{ Recuento } \\
\hline & & \multicolumn{2}{|c|}{ ¿Conoce la diabetes? } & \multirow[b]{2}{*}{ Total } \\
\hline & & Sí & No & \\
\hline \multirow[t]{5}{*}{ Edad } & 15 - 19 años & 7 & 3 & 10 \\
\hline & 20 - 29 años & 11 & 4 & 15 \\
\hline & 30 - 39 años & 7 & 3 & 10 \\
\hline & 40 - 49 años & 3 & 0 & 3 \\
\hline & $\begin{array}{l}60 \text { años en } \\
\text { adelante }\end{array}$ & 1 & 1 & 2 \\
\hline Total & & 29 & 11 & 40 \\
\hline
\end{tabular}




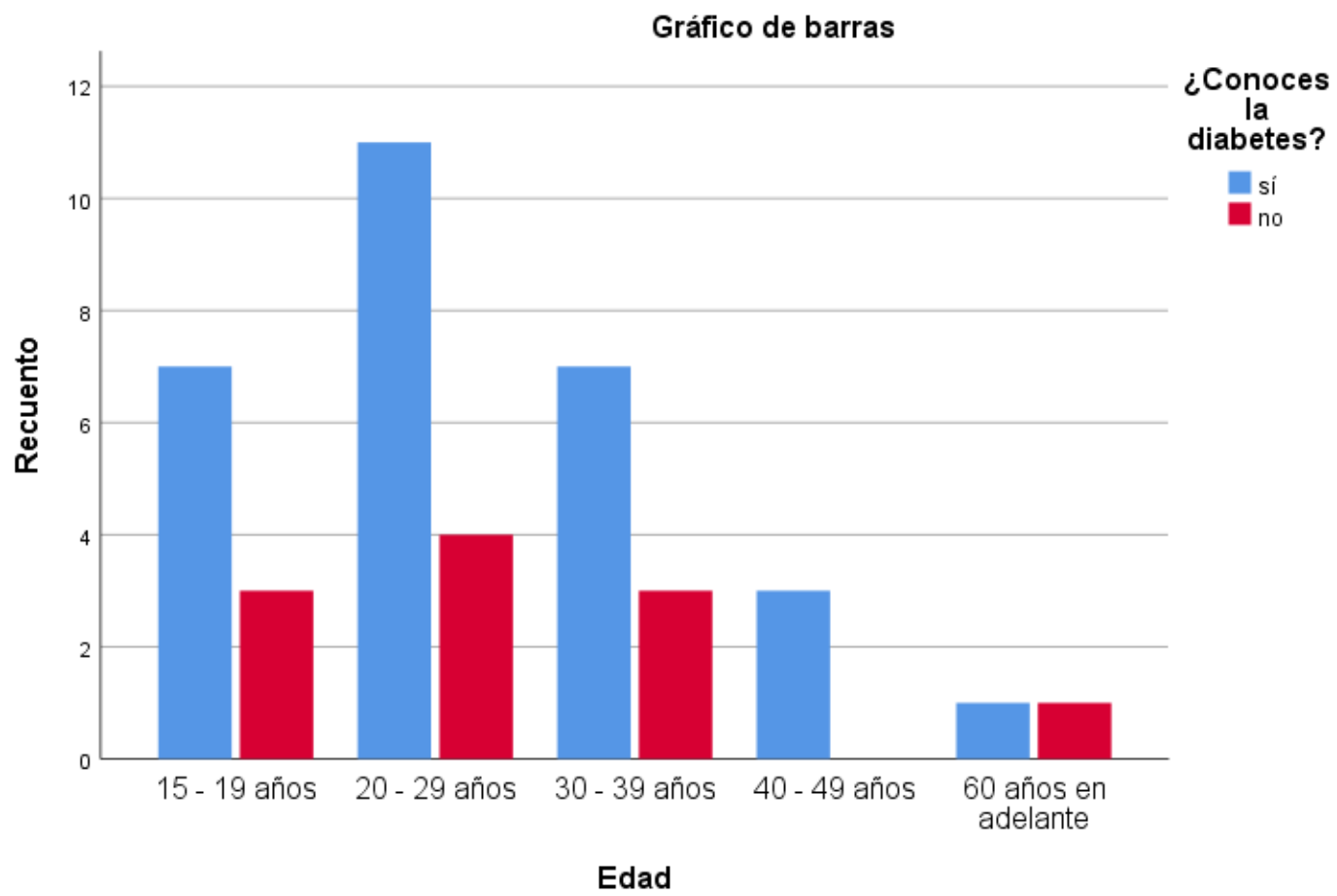

Enfocándonos en el conocimiento de la diabetes, de los 40 encuestados solo 29 conoce qué es la diabetes, siendo el $11 \%$ restante totalmente ignorantes sobre este tema, llegando a la conclusión del mal consumo de bebidas, ya que los jugos endulzados y la cola son las bebidas que están primeras en la lista en su dieta.

\section{Tabla cruzada Edad ${ }^{\star}$ Conoce a alguien cercano que tenga diabetes}

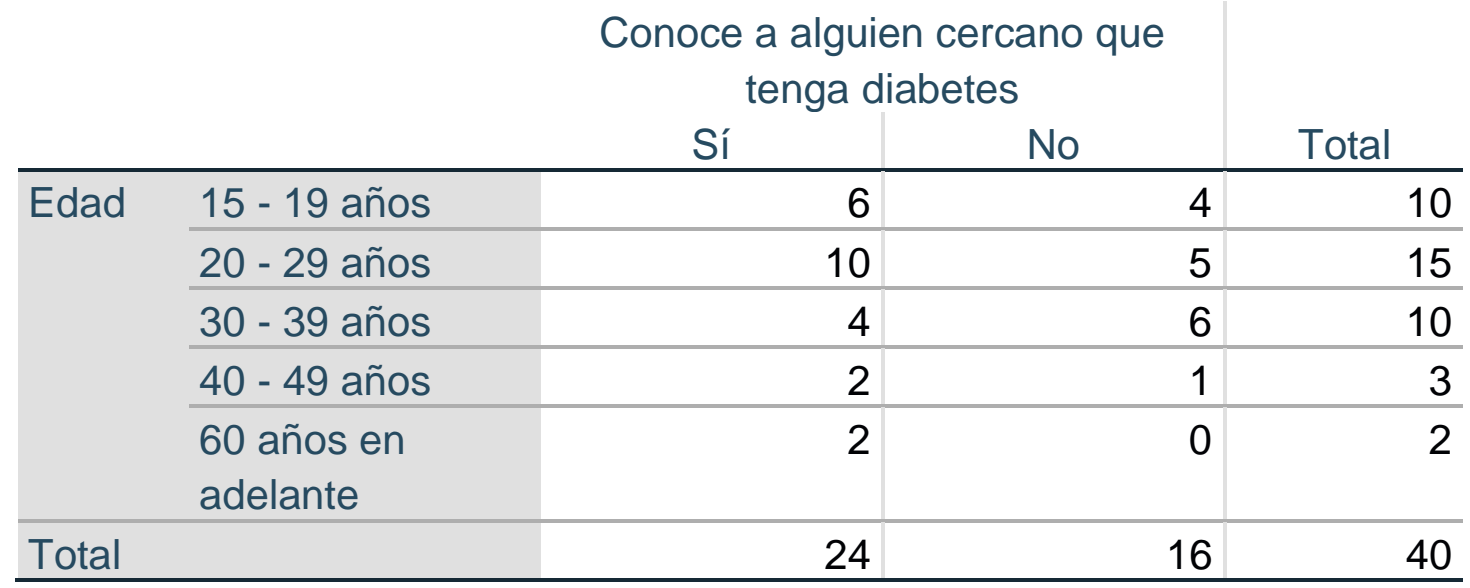




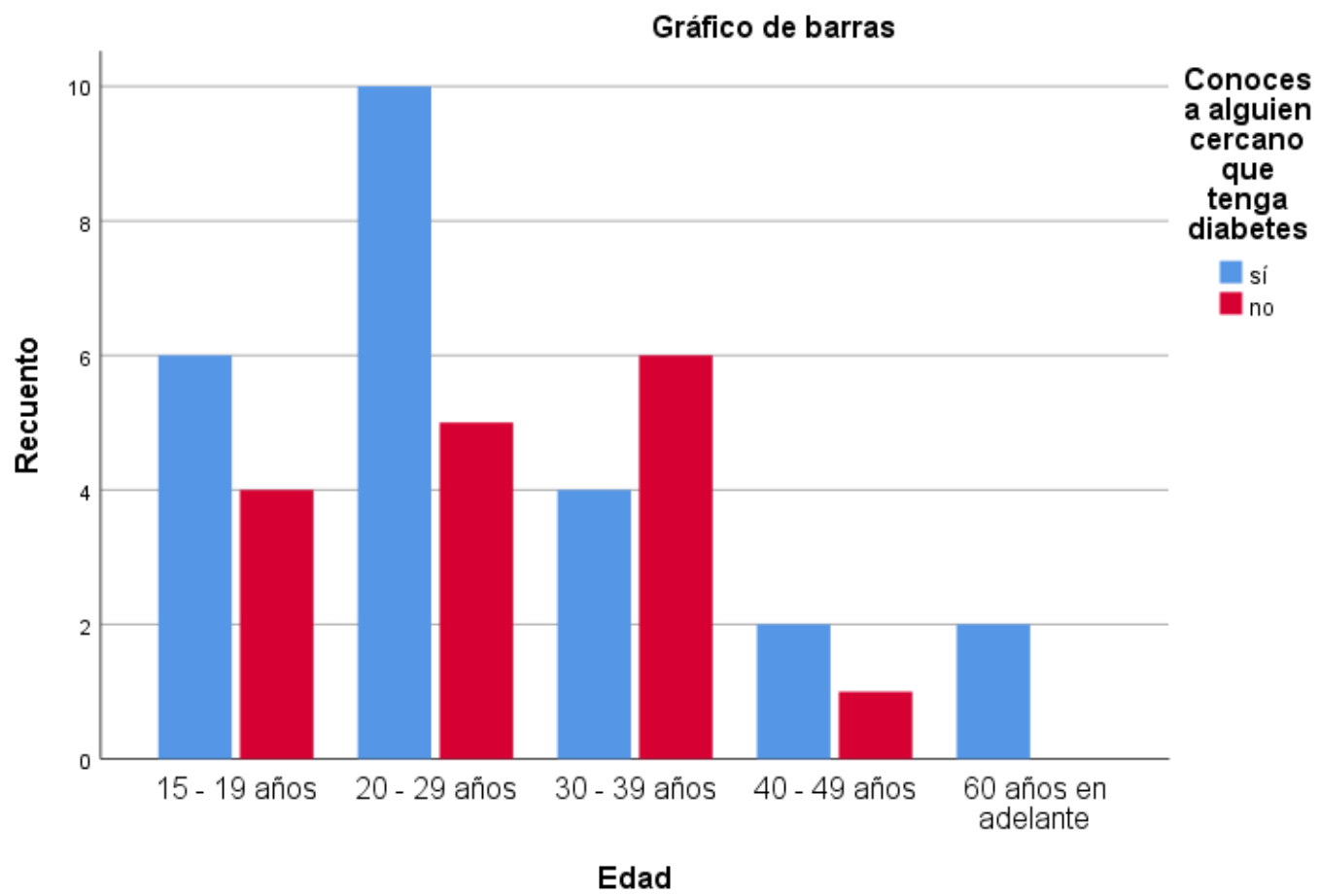

De los 40 encuestados 24 tienen a algún familiar, amigo o conocido, que padece diabetes, siendo este índice alto en el rango de personas con diabetes en la sociedad cuencana, mientras que 16 personas no conocen a nadie que la sufra, probablemente debido al alto índice de personas que no conocen qué es la diabetes.

Tabla cruzada Edad ${ }^{\star} ¿$ Sabe si se puede prevenir la diabetes?

\begin{tabular}{|c|c|c|c|c|}
\hline & & $\begin{array}{l}\text { ¿Sabe si se puec } \\
\text { diabetes? }\end{array}$ & enir la & \\
\hline & & Sí & No & Total \\
\hline Edad & $15-19$ años & 8 & 2 & 10 \\
\hline & $20-29$ años & 11 & 4 & 15 \\
\hline & 30 - 39 años & 2 & 8 & 10 \\
\hline & $40-49$ años & 2 & 1 & 3 \\
\hline & $\begin{array}{l}60 \text { años en } \\
\text { adelante }\end{array}$ & 1 & 1 & 2 \\
\hline Total & & 24 & 16 & 40 \\
\hline
\end{tabular}




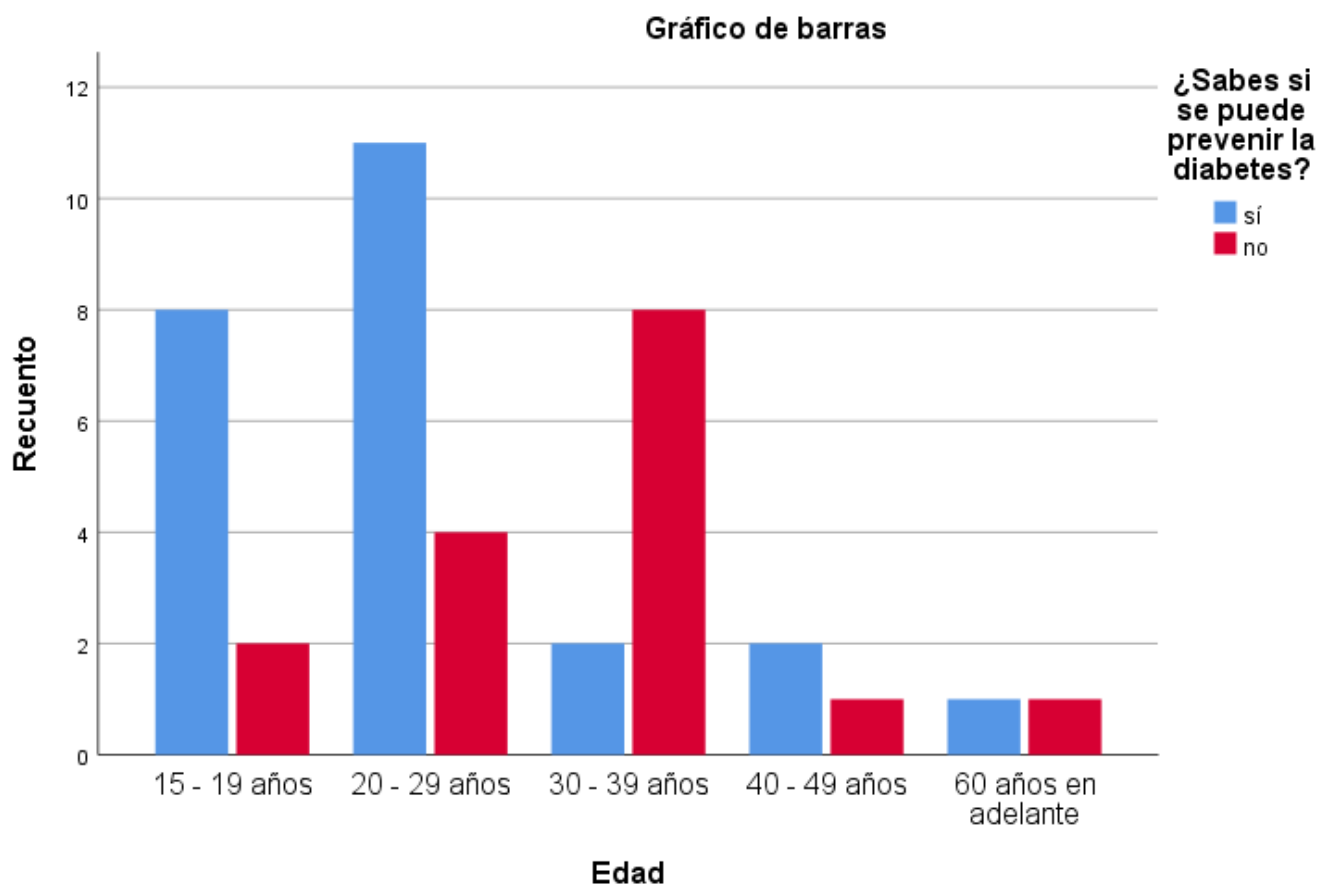

Tabla cruzada Edad ${ }^{\star} ¿$ Cree que en algún momento pueda llegar a tener diabetes?

Recuento

\begin{tabular}{|c|c|c|c|c|}
\hline & & \multicolumn{2}{|c|}{$\begin{array}{l}\text { ¿Cree que en algún momento } \\
\text { pueda llegar a tener diabetes? }\end{array}$} & \multirow[b]{2}{*}{ Total } \\
\hline & & Sí & No & \\
\hline \multirow[t]{5}{*}{ Edad } & 15 - 19 años & 5 & 5 & 10 \\
\hline & 20 - 29 años & 8 & 7 & 15 \\
\hline & 30 - 39 años & 7 & 3 & 10 \\
\hline & 40 - 49 años & 2 & 1 & 3 \\
\hline & $\begin{array}{l}60 \text { años en } \\
\text { adelante }\end{array}$ & 1 & 1 & 2 \\
\hline Total & & 23 & 17 & 40 \\
\hline
\end{tabular}




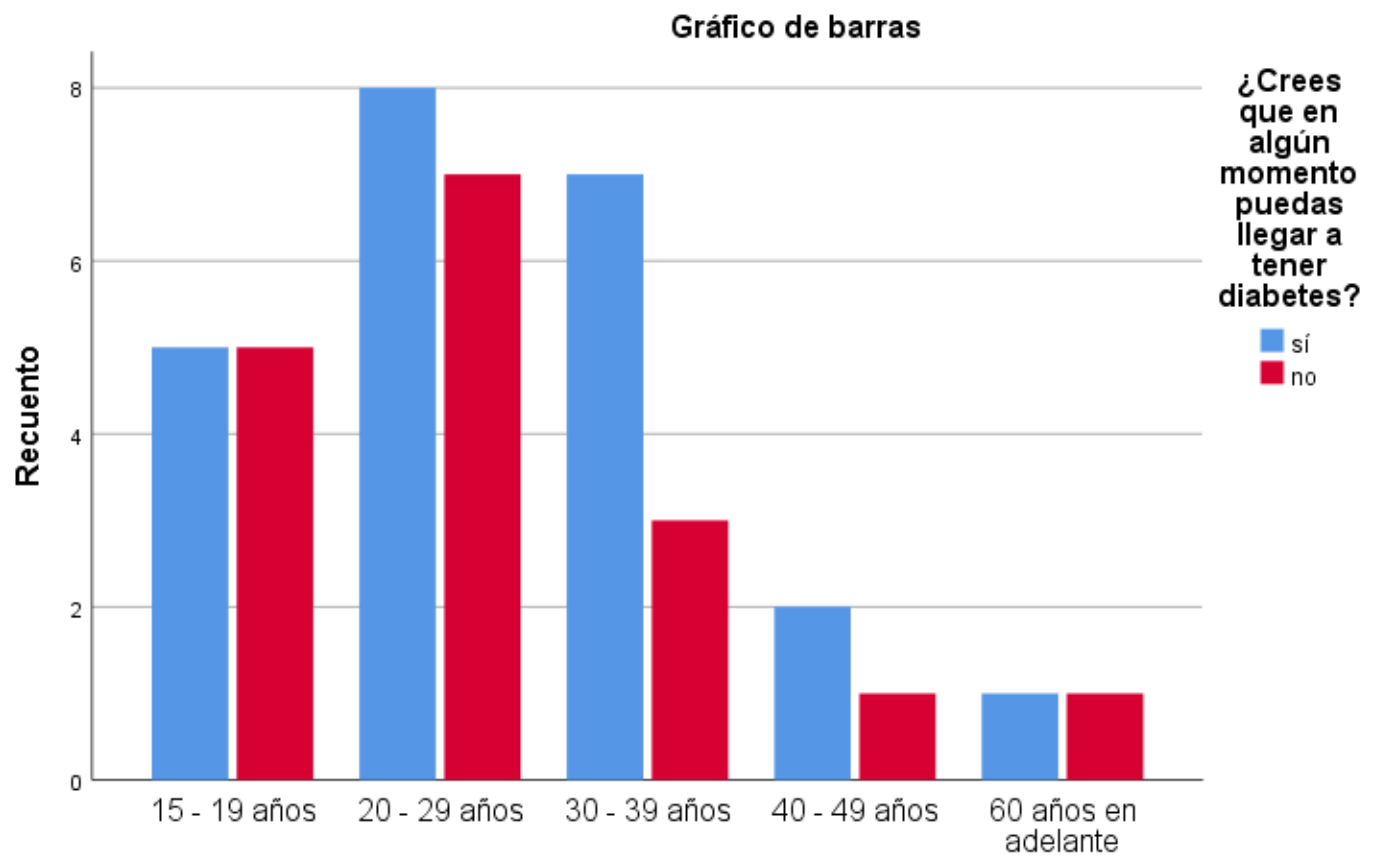

Edad

Con estas dos preguntas se puede contrastar aún más el conocimiento sobre la enfermedad, ya que se posee los mismos valores de las preguntas 9 y 10 y al mismo tiempo nos damos cuenta del porqué del desinterés en la alimentación y consumo de bebidas.

Tabla cruzada Edad* ¿Cree que es factible realizar una campaña de prevención sobre la diabetes? Recuento

\begin{tabular}{|c|c|c|c|}
\hline & & $\begin{array}{l}\text { ¿Cree que es } \\
\text { factible realizar } \\
\text { una campaña de } \\
\text { prevención } \\
\text { sobre la } \\
\text { diabetes? } \\
\qquad \text { Sí }\end{array}$ & Total \\
\hline \multirow[t]{5}{*}{ Edad } & 15 - 19 años & 10 & 10 \\
\hline & 20 - 29 años & 15 & 15 \\
\hline & 30 - 39 años & 10 & 10 \\
\hline & 40 - 49 años & 3 & 3 \\
\hline & $\begin{array}{l}60 \text { años en } \\
\text { adelante }\end{array}$ & 2 & 2 \\
\hline Total & & 40 & 40 \\
\hline
\end{tabular}




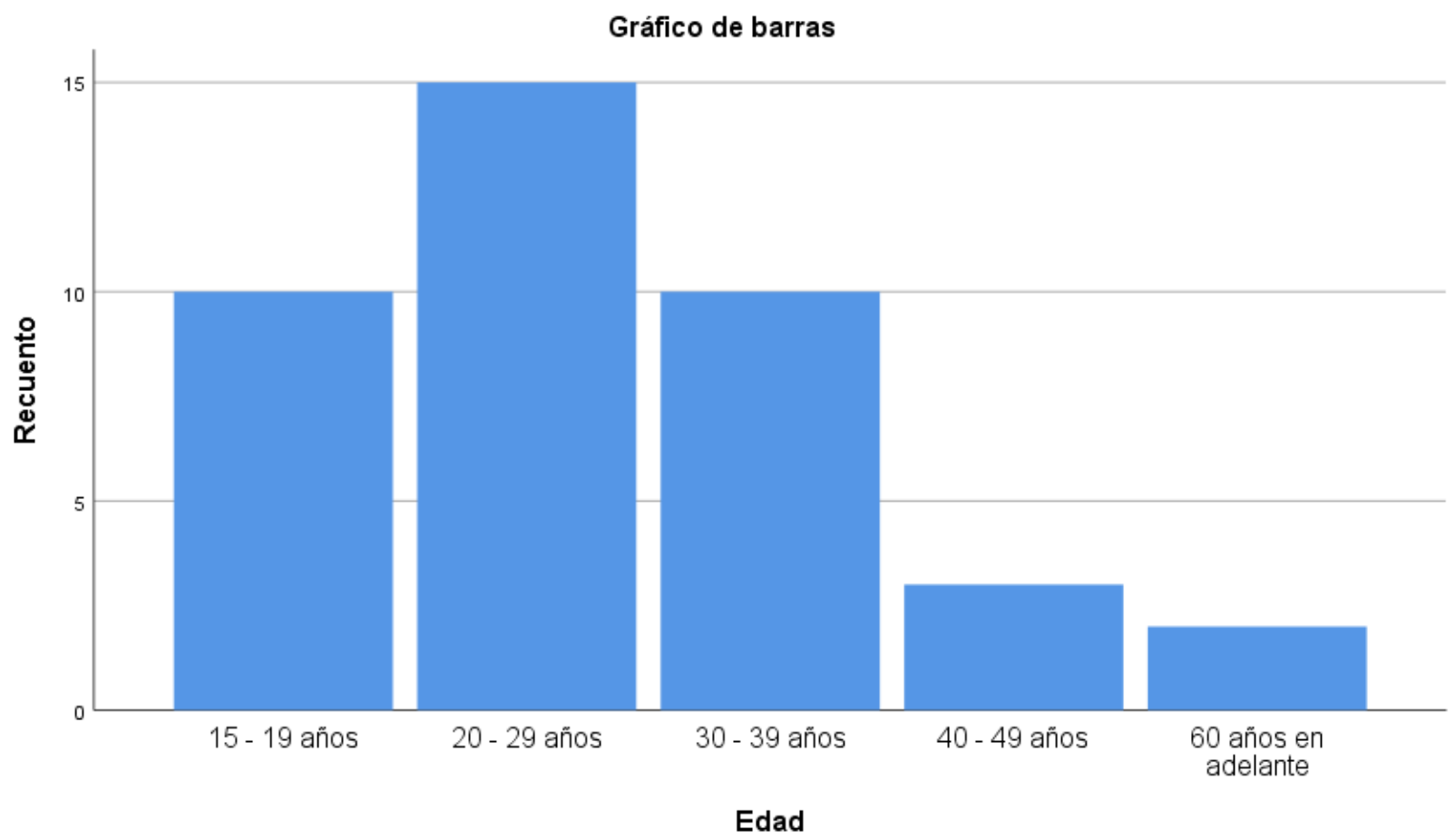

Tabla cruzada Edad ${ }^{\star} ¿ A$ qué tipo de gente cree que les beneficiaría la campaña de diabetes?

Recuento

¿A qué tipo de gente cree que les beneficiaría

la campaña de diabetes?

Madre de Universi-

Adultos

familia tarios

Niños

Todos

Total

\begin{tabular}{|c|c|c|c|c|c|c|c|}
\hline \multirow[t]{5}{*}{ Edad } & 15 - 19 años & 1 & 0 & 1 & 0 & 8 & 10 \\
\hline & 20 - 29 años & 2 & 0 & 0 & 1 & 12 & 15 \\
\hline & 30 - 39 años & 2 & 1 & 0 & 0 & 7 & 10 \\
\hline & 40 - 49 años & 2 & 0 & 0 & 0 & 1 & 3 \\
\hline & $\begin{array}{l}60 \text { años en } \\
\text { adelante }\end{array}$ & 1 & 0 & 0 & 0 & 1 & 2 \\
\hline Total & & 8 & 1 & 1 & 1 & 29 & 40 \\
\hline
\end{tabular}




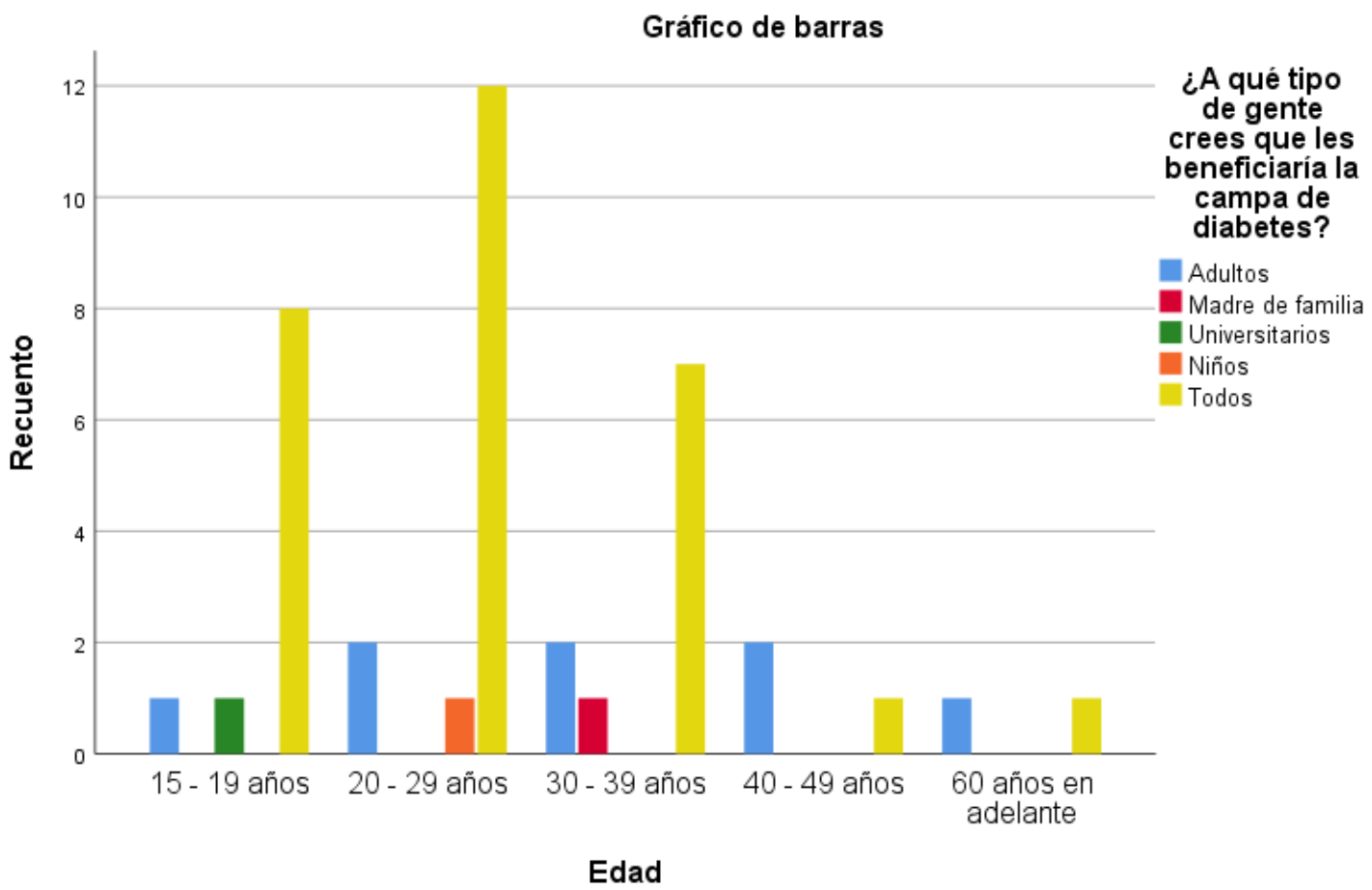

Al finalizar obtenemos como conclusión, que se causó una sensibilización por el tema y el $100 \%$ de los encuestados desean que se haga una campaña sobre la prevención y el conocimiento de esta enfermedad para todo el público.

De acuerdo a la investigación, la sociedad cuencana tiene una alta probabilidad de padecer diabetes, debido a su desinterés sobre dicha enfermedad, a que su estilo de alimentación es bastante malo -prefieren los jugos azucarados, las gaseosas, etc. a tomar agua natural-, y a que el ejercicio no lo realizan por salud, sino por verse bien en su mayoría. Es por esto, que se necesita educación sobre esta enfermedad, para disminuir el número de personas que pueda llegar a sufrirla en la sociedad cuencana y que no llegue a las demás generaciones debido al mal estilo de vida.

\section{DISCUSIÓN}

Se concluye que la diabetes es una enfermedad que tiene múltiples causas, como lo es, fundamentalmente, la mala alimentación y la predisposición genética a dicha enfermedad. La falta de educación que existe acerca de este tema y sobre todo la falta de interés en la población cuencana sobre la salud, al no alimentarse bien, no hacer ejercicio, no tomar agua en vez de jugos y gaseosas, etc.

Es importante tomar conciencia sobre esta enfermedad ya que está muy difundida y todos corren riesgos de contraerla aunque no se tenga predisposición genética a ella, ya que actualmente, existen alimentos que son consumidos excesivamente y la falta de ejercicio, pueden acercarnos a dicha enfermedad.

Todo lo mencionado antes, coincide con investigaciones como las de la Dra. Silvia Mayela Brove Urbina, médico pediatra, que expone en su tesis sobre los índices de personas con diabetes, el poco conocimiento que existe sobre la enfermedad haciendo que el índice aumente en las generaciones que vienen en camino, y 
plantea una campaña que marque la calidad de vida en la población que permita influir en las generaciones futuras para que lleven un estilo de vida saludable y sano.

Es responsabilidad de las autoridades de la Salud pública hacer campañas de orientación alimenticia para disminuir los índices de padecimiento de esta enfermedad. Con la presente investigación se ha obtenido una respuesta positiva sobre la educación de la diabetes en los encuestados, que se sienten interesados por el tema.

Además de presentar información de la enfermedad, ésta deberá ser la apropiada para que no cause más confusión en la sociedad. En muchos casos la información no fue transmitida de la manera apropiada y provocó ver esta dolencia como algo que es intratable, o como algo con lo que simplemente no se puede vivir, cuando debe ser todo lo contrario, ya que la diabetes es una enfermedad absolutamente controlable y evitable en aquellos casos en los que aún no se ha contraído.

\section{BIBLIOGRAFÍA}

Asociación Diabetes Madrid. (2015). Recuperado de https://diabetesmadrid.org/vivircon-diabetes-tipo2/

Canala Diabetes (24 de Enero de 2016). Recuperado de http://www.canaldiabetes.com/los-7-beneficios-de-tener-diabetes/

CDC. Centros para el Control y la Prevención de Enfermedades. (14 de Abril de 2015). Recuperado de https://www.cdc.gov/diabetes/spanish/basics/diabetes.html

Diabetes, Bienestar \& Salud. (2014). Recuperado de https://www.diabetesbienestarysalud.com/guia-diabetes/que-es-diabetes/2013/02/

La Vanguardia (17 de Marzo de 2012). Especialistas y médicos comparten los nuevos avances sobre la diabetes. Recuperado de http://www.lavanguardia.com/salud/20120417/54284863361/especialistasmedicos-avances-diabetes.html

Mariano, S. (2014). Diabetes, Bienestar \& Salud . Recuperado de https://www.diabetesbienestarysalud.com/expertos/ayudar-sin-fastidio/2017/10/ http://www.salud.gob.mx/unidades/cdi/documentos/diabetes_mellitus.pdf http://www.antorres.com/2011/05/5-pasos-para-crear-una-campana-de-social-media/ https://ecosas.wordpress.com/2006/11/16/10-pasos-para-hacer-campanaspublicitarias/ 